\title{
Charm Physics at B-factories
}

\author{
Long-Ke Li*i (On behalf of the Belle Collaboration) \\ Institute of High Energy Physics, CAS, 100049 China \\ E-mail: rilongkedihep.ac.cn
}

Belle and $\mathrm{BaBar}$ have achieved fruitful results on charm physics at B-factories, based on datasets of an integrated luminosity of about $988 \mathrm{fb}^{-1}$ and $518 \mathrm{fb}^{-1}$, respectively. In this proceedings, some recent measurements on $D^{0}-\bar{D}^{0}$ mixing, $C P$ violation and charmed baryons are reported. The $D^{0}-\bar{D}^{0}$ mixing parameters in $D^{0} \rightarrow \pi^{+} \pi^{-} \pi^{0}$ are first measured at the BaBar experiment: $x=(1.5 \pm 1.2 \pm 0.6) \%$ and $y=(0.2 \pm 0.9 \pm 0.5) \%$. A first measurement of $T$-odd moments in $D^{0} \rightarrow K_{S}^{0} \pi^{+} \pi^{-} \pi^{0}$ decays is performed at the Belle experiment: $a_{C P}=\left(-0.28 \pm 1.38_{-0.76}^{+0.23}\right) \times 10^{-3}$ which is consistent with no $C P$ violation. Several studies on the charmed baryons at the Belle experiment are reported, including a search for a hidden-strange pentaquark $P_{s}^{+}$in $\Lambda_{c}^{+} \rightarrow \phi p \pi^{0}$; a first observation of $\Omega_{c}(2930)^{0}$ in $B^{-} \rightarrow K^{-} \Lambda_{c}^{+} \bar{\Lambda}_{c}^{-}$; a measurements of branching fractions of eight $\Omega_{c}$ hadronic decay modes and a confirmation of four of the five narrow excited $\Omega_{c}$ states reported by the LHCb experiment in $\Xi_{c}^{+} K^{-}$invariant mass: the $\Omega_{c}(3000), \Omega_{c}(3050), \Omega_{c}(3066)$ and $\Omega_{c}(3090)$; the inclusive production cross sections of hyperons and charmed baryons for $e^{+} e^{-}$ annihilation near $\Upsilon(4 S)$.

The International Conference on B-Physics at Frontier Machines - BEAUTY2018

6-11 May, 2018

La Biodola, Elba Island, Italy

\footnotetext{
* Speaker.

${ }^{\dagger}$ Supported in part by National Natural Science Foundation of China (NSFC) under contract Nos. 11475187 and 11521505; Key Research Program of Frontier Sciences, CAS, Grant No. QYZDJ-SSW-SLH011; and the CAS Center for Excellence in Particle Physics (CCEPP).
} 


\section{Machines and charm dataset}

The Belle detector at KEKB and the BABAR detector at PEP-II have collected an integrated luminosity of about $988 \mathrm{fb}^{-1}$ and $518 \mathrm{fb}^{-1}$, respectively, mainly at the energy of the $\Upsilon(4 S)$ resonance with asymmetric $e^{+} e^{-}$energy collisions. $\Upsilon(4 S)$ decays to $B \bar{B}$ pairs (so-called B-factories). These two detectors have good momentum resolution and vertex resolution. A detailed description is available in Ref. [四. Besides as B-factories, KEKB and PEP-II are also $\tau$-charm factories where a large available charm sample is produced and it has a good reconstruction efficiency. The available charm samples at several experiments in different kinds of colliders are summarised in Table $\mathbb{0}[\mathbf{[}]$. In this table, the charm sample $N$ does not include charmed hadrons from $B$ decays at B-factories, where a large charm sample from $B$ mesons via $b \rightarrow c$ transition is also available to study charm physics.

Table 1: Available charm dataset at some experiments at different kinds of colliders.

\begin{tabular}{|c|c|c|c|c|c|c|}
\hline Experiment & Machine & C.M $\sqrt{s}$ & Luminosity & charm sample & efficiency & (-)advantage/ $\odot$ disadvantage \\
\hline \multirow{5}{*}{$\begin{array}{c}\text { CLEOc }= \\
=0= \\
=\end{array}$} & $\begin{array}{l}\text { CESR } \\
\left(e^{+} e^{-}\right)\end{array}$ & $3.77 \mathrm{GeV}$ & $0.8 \mathrm{fb}^{-1}$ & $\begin{array}{l}2.9 \times 10^{6}\left(D^{0}\right) \\
2.3 \times 10^{6}\left(D^{+}\right)\end{array}$ & \multirow{5}{*}{$\sim 10-30 \%$} & $\begin{array}{l}\text { () extremely clean enviroment } \\
\text { () pure D-beam, almost no bkg }\end{array}$ \\
\hline & \multirow{4}{*}{$\begin{array}{c}\text { BEPC-II } \\
\left(e^{+} e^{-}\right)\end{array}$} & $4.17 \mathrm{GeV}$ & $0.6 \mathrm{fb}^{-1}$ & $0.6 \times 10^{6}\left(D_{s}^{+}\right)$ & & \multirow{4}{*}{$\begin{array}{l}\text { () pure D-beam, almost no bkg } \\
\text { () quantum coherence } \\
\text { () no CM boost, no T-dep analyses }\end{array}$} \\
\hline & & $3.77 \mathrm{GeV}$ & $2.9 \mathrm{fb}^{-1}$ & $\begin{array}{l}10.5 \times 10^{6}\left(D^{0}\right) \\
8.4 \times 10^{6}\left(D^{+}\right)\end{array}$ & & \\
\hline & & $4.18 \mathrm{GeV}$ & $3.0 \mathrm{fb}^{-1}$ & $3 \times 10^{6}\left(D_{s}^{+}\right)$ & & \\
\hline & & $4.6 \mathrm{GeV}$ & $0.6 \mathrm{fb}^{-1}$ & $\begin{array}{c}1 \times 10^{5}\left(\Lambda_{c}^{+}\right) \\
\star\end{array}$ & & \\
\hline \multirow{11}{*}{$\sum_{B E L L E}$} & \multirow{9}{*}{$\begin{array}{l}\text { PEP-II } \\
\left(e^{+} e^{-}\right)\end{array}$} & \multirow{5}{*}{$10.58 \mathrm{GeV}$} & \multirow{5}{*}{$1 a b^{-1}$} & $1.3 \times 10^{9}\left(D^{0}\right)$ & \multirow{8}{*}{$\sim 5-10 \%$} & \multirow{9}{*}{ 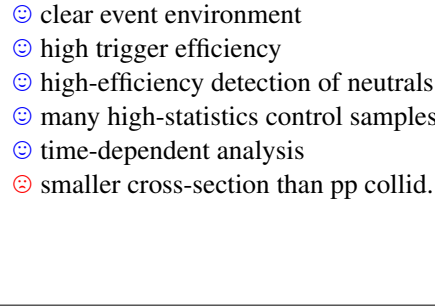 } \\
\hline & & & & $7.7 \times 10^{8}\left(D^{+}\right)$ & & \\
\hline & & & & $2.5 \times 10^{8}\left(D_{s}^{+}\right)$ & & \\
\hline & & & & $1.5 \times 10^{8}\left(\Lambda_{c}^{+}\right)$ & & \\
\hline & & & & $6.5 \times 10^{8}\left(D^{0}\right)$ & & \\
\hline & & \multirow{4}{*}{$10.58 \mathrm{GeV}$} & \multirow{4}{*}{$0.5 a b^{-1}$} & $3.8 \times 10^{8}\left(D^{+}\right)$ & & \\
\hline & & & & $1.2 \times 10^{8}\left(D_{s}^{+}\right)$ & & \\
\hline & & & & $0.7 \times 10^{8}\left(\Lambda_{c}^{+}\right)$ & & \\
\hline & & & & $\star \star$ & \multirow[t]{2}{*}{$\star \star$} & \\
\hline & $\begin{array}{l}\text { Tevatron } \\
\qquad(p \bar{p})\end{array}$ & $1.96 \mathrm{TeV}$ & $9.6 \mathrm{fb}^{-1}$ & $1.3 \times 10^{11}$ & & $\begin{array}{l}\text { () large production cross-section } \\
\text { (-) large boost }\end{array}$ \\
\hline & & & & & \multirow{2}{*}{$<0.5 \%$} & (-) excellent time resolution \\
\hline \multirow{2}{*}{$\begin{array}{l}\text { LHCb } \\
\text { THAp }\end{array}$} & $\begin{array}{l}\mathrm{LHC} \\
(p p)\end{array}$ & $8 \mathrm{TeV}$ & $\begin{array}{l}1.0 \mathrm{fb}^{-1} \\
2.0 \mathrm{fb}^{-1}\end{array}$ & $5.0 \times 10^{12}$ & & \multirow{2}{*}{$\begin{array}{l}\text { () dedicated trigger required } \\
\text { (-) hard to do with neutrals/neutrinos }\end{array}$} \\
\hline & & & & $\star \star \star$ & $\star$ & \\
\hline
\end{tabular}

\section{2. $D^{0}-\bar{D}^{0}$ mixing and $C P$ violation}

Since $D^{0}-\bar{D}^{0}$ mixing, as the only up-type quark meson mixing, has already been observed with a confidence level of more than $5 \sigma$ in a single decay channel [ [3], 团], all mixing phenomena of the open-flavor neutral mesons, which originate from the difference between the flavor and mass eigenstates of the meson-anti-meson system, are well established. The two mass eigenstates $D_{1}$ and $D_{2}$ of the effective Hamiltonian matrix $\mathscr{H}=\left(M-\frac{i}{2} \Gamma\right)$ are given by the mixture of flavoreigenstates: $\left|D_{1,2}\right\rangle=p\left|D^{0}\right\rangle \pm q\left|\bar{D}^{0}\right\rangle$, here $|p|^{2}+|q|^{2}=1$ if CPT is conserved, and we assume $C P\left|D^{0}\right\rangle=\left|\bar{D}^{0}\right\rangle .\left|D_{1}\right\rangle$ is the positive-CP eigenstate and $\left|D_{2}\right\rangle$ is the negative-CP eigenstate, assuming no CP violation. The mixing parameters, $x=\Delta m / \bar{\Gamma}$ and $y=\Delta \Gamma /(2 \bar{\Gamma})$, are related to the mass and 
the width differences $\Delta m$ and $\Delta \Gamma$ between the two mass eigenstates and $\bar{\Gamma}$ is the average decay width of the mass eigenstates. The mixing parameters $x$ and $y$ are difficult to calculate. The Standard Model (SM) predicts that $D^{0}-\bar{D}^{0}$ mixing can occur via short distance effects and long distance effects and is strongly suppressed to $\sim 1 \%$.

There are three types of CP violation (CPV) according to their different sources: (1) in the mixing (indirect CPV): $r_{m}=|q / p| \neq 1$; (2) in the decay (direct CPV): $\left|\bar{A}_{\bar{f}} / A_{f}\right| \neq 1$, Here the amplitudes of $D^{0}$ decays are defined as: $\left\langle f|\mathscr{H}| D^{0}\right\rangle=A_{f},\left\langle\bar{f}|\mathscr{H}| \bar{D}^{0}\right\rangle=\bar{A}_{\bar{f}}$; (3) in the interference between mixing and decay: $\arg (q / p) \neq 0$.

\section{1 $D^{0}-\bar{D}^{0}$ mixing in $D^{0} \rightarrow \pi^{+} \pi^{-} \pi^{0}$}

The status of $D^{0}-\bar{D}^{0}$ mixing results from charm factories, B-factories and hadron colliders are available from the Heavy Flavor Averaging Group (HFLAV) [5]. These measurements have excluded the no-mixing hypothesis with more than $11.5 \sigma$ confidence level, assuming CPV is allowed. Many of them are reported by the Belle and BaBar experiments at B-factories. Only one (three) decay channels have reached the observation (evidence) confidence level. More decay channels or larger samples need to be studied at different experiments.

Recently, BaBar performed the first measurement on the $D^{0}-\bar{D}^{0}$ mixing parameters using a time-dependent Dalitz analysis of the decay $D^{0} \rightarrow \pi^{+} \pi^{-} \pi^{0}[$ [G], based on a dataset with an integrated luminosity of $468.1 \mathrm{fb}^{-1}$, shown in Fig. $\square$. The measured mixing parameters are $x=$ $(1.5 \pm 1.2 \pm 0.6) \%$ and $y=(0.2 \pm 0.9 \pm 0.5) \%$, where the quoted uncertainties are statistical and systematic, respectively. Considering this result is statistically limited, this analysis is anticipated at Belle II experiment with about 100 times the BaBar dataset.

\section{2 $T$-odd asymmetry in $D^{0} \rightarrow K_{S}^{0} \pi^{+} \pi^{-} \pi^{0}$}

A first measurement of the $T$-odd moments in $D^{0} \rightarrow K_{S}^{0} \pi^{+} \pi^{-} \pi^{0}$ is reported at Belle based on a data sample with integrated luminosity $966 \mathrm{fb}^{-1}[\square]$. The $C P$-violation-sensitive asymmetry is defined as $a_{C P}^{\mathrm{T}-\text { odd }}=\frac{1}{2}\left(A_{T}-\bar{A}_{T}\right)$, where $A_{T}$ is the parity-odd asymmetry $A_{T}=\frac{\Gamma\left(C_{T}>0\right)-\Gamma\left(C_{T}<0\right)}{\Gamma\left(C_{T}>0\right)+\Gamma\left(C_{T}<0\right)}$, where $C_{T}$ is the parity-odd observable shown in Fig. $\mathbb{Z}$ (left). The result is $a_{C P}^{T-d d}=(-0.28 \pm$ $\left.1.38_{-0.76}^{+0.23}\right) \times 10^{-3}$, which is no $C P$ violation. This result constitutes one of the most precise tests of $C P$ violation in the $D$ meson system. The measurement uncertainties are statistically dominated and thus can be improved further with the data from the upcoming Belle II experiment. In addition, the $a_{C P}^{T-o d d}$ measurements in different regions of this decay phase space are performed and are also consistent with no $C P$ violation.

\section{3 $C P$ asymmetry in $D^{+} \rightarrow \pi^{+} \pi^{0}$}

Singly-Cabibbo-suppressed decays are excellent candidates to probe $C P$ violation in the charm sector. Any $C P$ asymmetry found in these channels points to New Physics. Based on the dataset of $921 \mathrm{fb}^{-1}$ collected with the Belle detector, $C P$ asymmetries are measured via a simultaneous fit to the invariant mass of the $D$ meson in the decay $\left.D^{+} \rightarrow \pi^{+} \pi^{0}[]\right]: A_{\text {raw }}^{\pi \pi}=\frac{N\left(D^{+} \rightarrow \pi^{+} \pi^{0}\right)-N\left(D^{-} \rightarrow \pi^{-} \pi^{0}\right)}{N\left(D^{+} \rightarrow \pi^{+} \pi^{0}\right)+N\left(D^{-} \rightarrow \pi^{-} \pi^{0}\right)}=$ $A_{C P}+A_{F B}+A_{\varepsilon}^{\pi^{ \pm}}$, and obtain $A_{\text {raw }}^{\pi \pi}=(+0.52 \pm 1.92) \%$ for a tagged $D$ sample and $A_{\text {raw }}^{\pi \pi}=(+3.77 \pm$ $1.60) \%$ for an untagged $D$ sample. The decay $D^{+} \rightarrow K_{S}^{0} \pi^{+}$is chosen as the normalisation channel, where the $C P$ asymmetry has been measured to be $A_{C P}^{K \pi}=(-0.363 \pm 0.094 \pm 0.067) \%$ [Q], where 

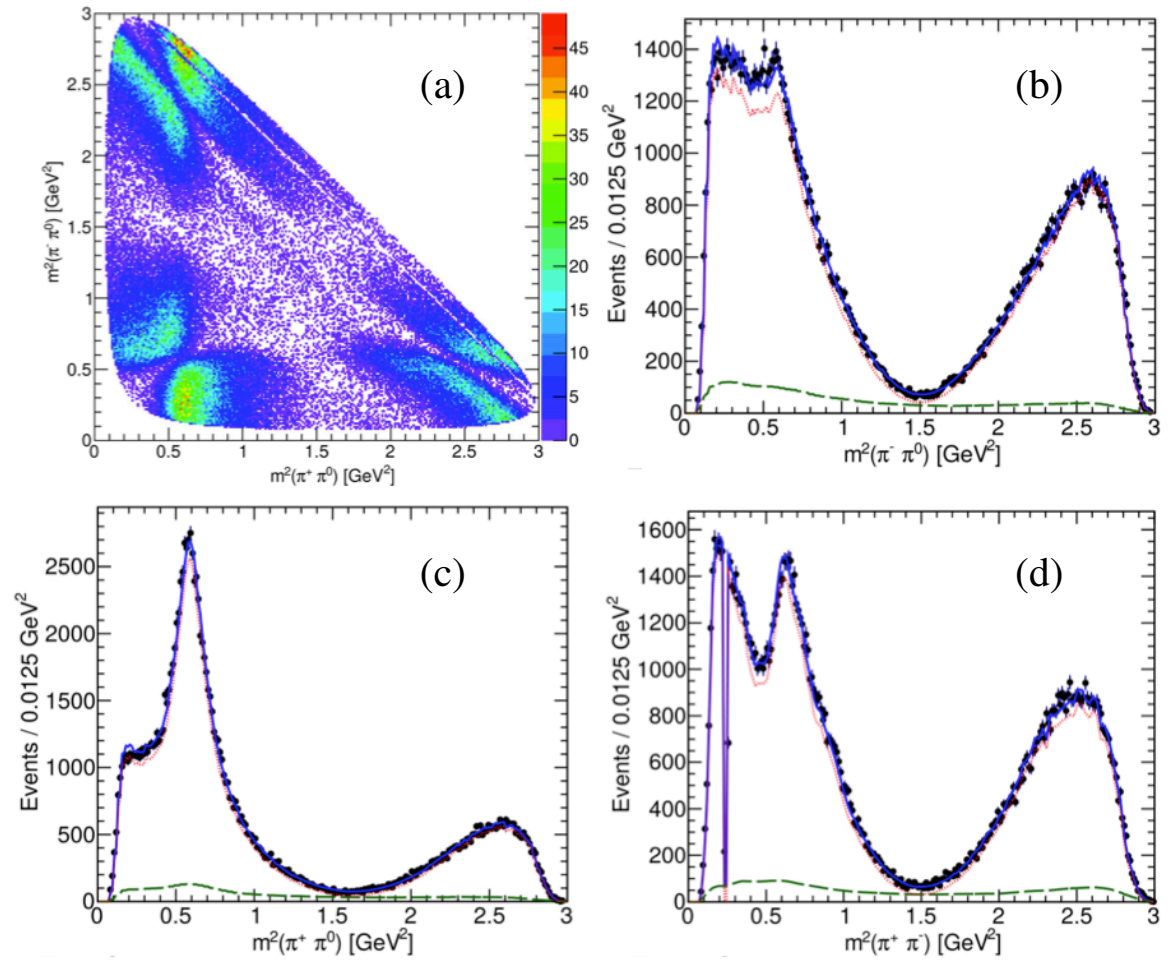

Figure 1: The Dalitz plot (a) and the Dalitz variables projections for $m_{\pi^{-} \pi^{0}}^{2}$ (b), $m_{\pi^{+} \pi^{0}}^{2}$ (c) and $m_{\pi^{+} \pi^{-}}^{2}$ (d) with the time-dependent Dalitz fitting result.
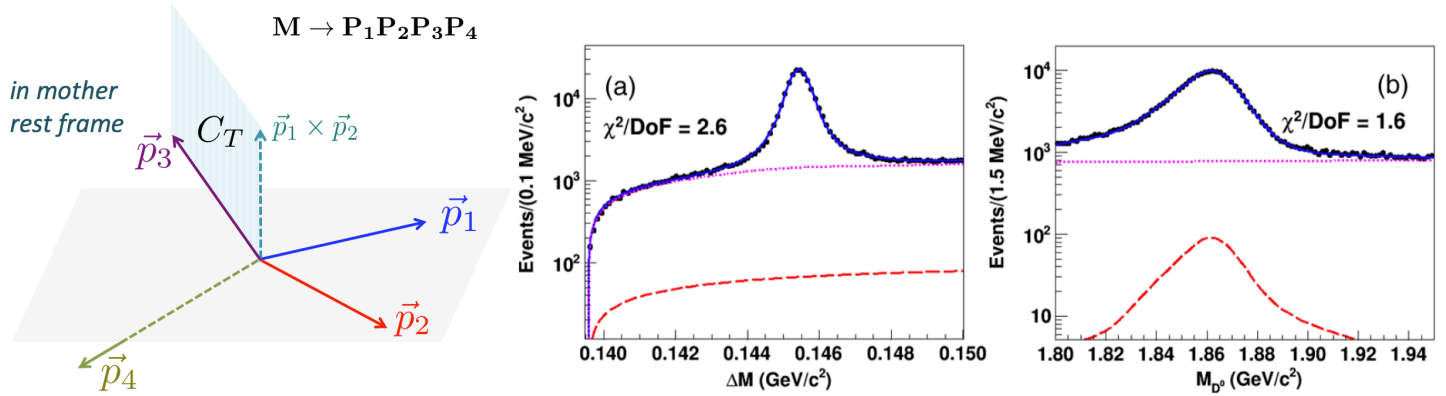

Figure 2: A schematic diagram for the definition of the parity-odd observable $C_{T}=\left(\vec{p}_{1} \times \vec{p}_{2}\right) \cdot \vec{p}_{3}$. One signal yields extraction via 2D fit on mass difference of $D^{*}$ and $D^{0}, \Delta M(\mathrm{a})$, and invariant mass of reconstructed $D, M_{D^{0}}(\mathrm{~b})$.

$A_{\text {raw }}^{K \pi}=(-0.29 \pm 0.44) \%$ for the tagged sample and $A_{\text {raw }}^{K \pi}=(-0.25 \pm 0.17) \%$ for the untagged sample. Combining the results, $\Delta A_{\text {raw }}=(+2.67 \pm 1.24 \pm 0.20) \%$ leads to $A_{C P}^{\pi \pi}=A_{C P}^{K \pi}+\Delta A_{\text {raw }}$, and $A_{C P}\left(D^{+} \rightarrow \pi^{+} \pi^{0}\right)$ is determined to be $(+2.31 \pm 1.24 \pm 0.23) \%$.

\section{Charmed Baryons spectroscopy and decays}

As a characteristic of charmed baryons, the di-quark structure in light quarks is different from the light flavor baryons because the mass of the charm quark is much larger than that of the light 
flavor quarks $(\mathrm{u}, \mathrm{d}, \mathrm{s})$. Even though we know there are three diagrams at tree level, external-W, internal-W and $\mathrm{W}$-exchange processes, contributing to charmed baryon weak decays, the strength of each process is not known. At B-factories, the ground state charmed baryons: $\Lambda_{c}, \Sigma_{c}, \Xi_{c}$ and $\Omega_{c}$ and their excited states are studied. B-factories also provide a good laboratory to study strange baryons via the $c \rightarrow s$ transition.

\subsection{Search for $\Lambda_{c}^{+} \rightarrow \phi p \pi^{0}$ and measurement of $\mathscr{B}\left(\Lambda_{c}^{+} \rightarrow K^{-} \pi^{+} p \pi^{0}\right)$}

The Cabibbo-suppressed decay $\Lambda_{c}^{+} \rightarrow \phi p \pi^{0}$ in $e^{+} e^{-}$collisions is searched for at Belle, based on a data sample of integrated luminosity $915 \mathrm{fb}^{-1}$ [ [0]]. The signal yield is determined to be $148 \pm 62$ from a $2 \mathrm{D}$ fit on $M\left(K^{+} K^{-} p \pi^{0}\right)$ and $M\left(K^{+} K^{-}\right)$. No significant signals are observed, shown in Figs. B(a) and (b), and an upper limit on the branching fraction is set to be $\mathscr{B}\left(\Lambda_{c}^{+} \rightarrow\right.$ $\left.\phi p \pi^{0}\right)<15.3 \times 10^{-5}$ at $90 \%$ confidence level. An intermediate hidden-strangeness pentaquark decay $P_{s}^{+} \rightarrow \phi p$ is sought. Insufficient evidence is found for this intermediate decay with signal yields $70 \pm 28$ shown in Fig. B(c), and the upper limit on the product branching fraction is set to be $\mathscr{B}\left(\Lambda_{c}^{+} \rightarrow P_{s}^{+} \pi^{0}\right) \times \mathscr{B}\left(P_{s}^{+} \rightarrow \phi p\right)<8.3 \times 10^{-5}$ at $90 \%$ confidence level. The branching fraction for the Cabibbo-favored decay $\Lambda_{c}^{+} \rightarrow K^{-} \pi^{+} p \pi^{0}$ is updated with the most precise measurement to date, $\mathscr{B}\left(\Lambda_{c}^{+} \rightarrow K^{-} \pi^{+} p \pi^{0}\right)=(4.42 \pm 0.05 \pm 0.12 \pm 0.16) \%$, which is consistent with recent BESIII result $(4.53 \pm 0.23 \pm 0.30) \%$ [ए]].
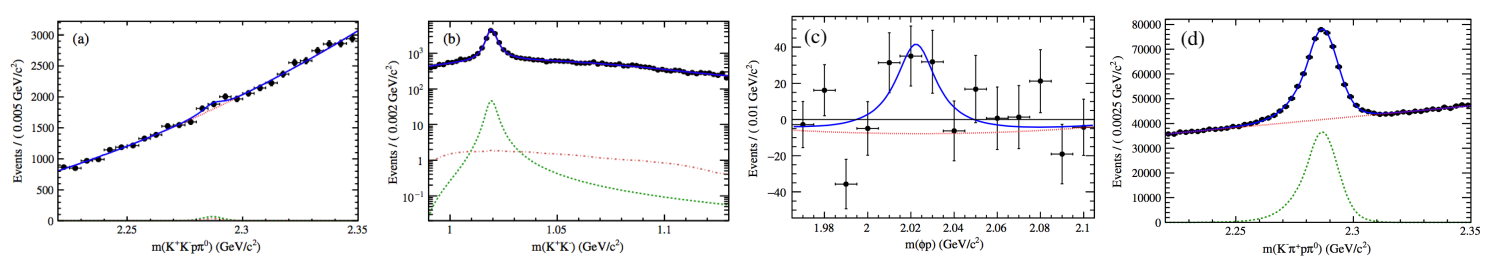

Figure 3: The projections of 2D fit on (a) $M\left(K^{+} K^{-} p \pi^{0}\right)$ and (b) $M\left(K^{+} K^{-}\right)$. The figure (c) is the backgroundsubtracted distribution of $m(\phi p)$ in $\phi p \pi^{0}$ final state. The figure (d) is the fitting to the invariant mass distribution of $m\left(K^{-} \pi^{+} p \pi^{0}\right)$.

\subsection{Observation of $\Xi_{c}(2930)^{0}$ in $B^{-} \rightarrow K^{-} \Lambda_{c}^{+} \bar{\Lambda}_{c}^{-}$}

The first observation of the $\Xi_{c}(2930)^{0}$ charmed-strange baryon with a significance $5.1 \sigma$ is reported in the invariant mass of $K^{-} \Lambda_{c}^{+}$in the decays $B^{-} \rightarrow K^{-} \Lambda_{c}^{+} \bar{\Lambda}_{c}^{-}$based on 772 million $B \bar{B}$ pairs at Belle [ㄹ] $]. \Lambda_{c}$ is reconstructed via five decay channels, $\Lambda_{c}^{+} \rightarrow p K^{-} \pi^{+}, p K_{S}^{0}, \Lambda \pi^{+}, p K_{S}^{0} \pi^{+} \pi^{-}$and $\Lambda \pi^{+} \pi^{+} \pi^{-}$. The $B$ meson candidates are extracted via a $2 \mathrm{D}$ fit on the beam-energy constrained mass $M_{b c}$ and the difference between the reconstructed $B$ invariant mass and nominal mass, $\Delta M_{B}$. The branching fraction of $B^{-} \rightarrow K^{-} \Lambda_{c}^{+} \bar{\Lambda}_{c}^{-}$is $(4.8 \pm 0.43 \pm 0.60) \times 10^{-4}$ which is consistent with world average $(6.9 \pm 2.2) \times 10^{-4}$ but with much improved precision. The mass and width of $\Xi_{c}(2930)^{0}$ are measured as $M=\left(2928.9 \pm 2.0_{-12.0}^{+0.9}\right) \mathrm{MeV} / c^{2}$, and $\Gamma=\left(19.5 \pm 8.4_{-7.9}^{+5.9}\right) \mathrm{MeV}$ shown in Fig. 团. No significant signals are seen in the $\Lambda_{c} \bar{\Lambda}_{c}^{-}$mass spectrum and an upper limit for $Y(4600)$ is set to be $\mathscr{B}\left(B^{-} \rightarrow K^{-} Y(4600)\right) \mathscr{B}\left(Y(4600) \rightarrow \Lambda_{c}^{+} \bar{\Lambda}_{c}^{-}\right)<1.2 \times 10^{-4}$.

\subsection{Branching ratios of $\Omega_{c}$ hadronic decays at Belle}

Because of constructive interference, the $\Omega_{c}$ baryon has the same flavor light quarks (ss) and a shorter lifetime compared with other ground state charmed baryons. Precise measurements of 

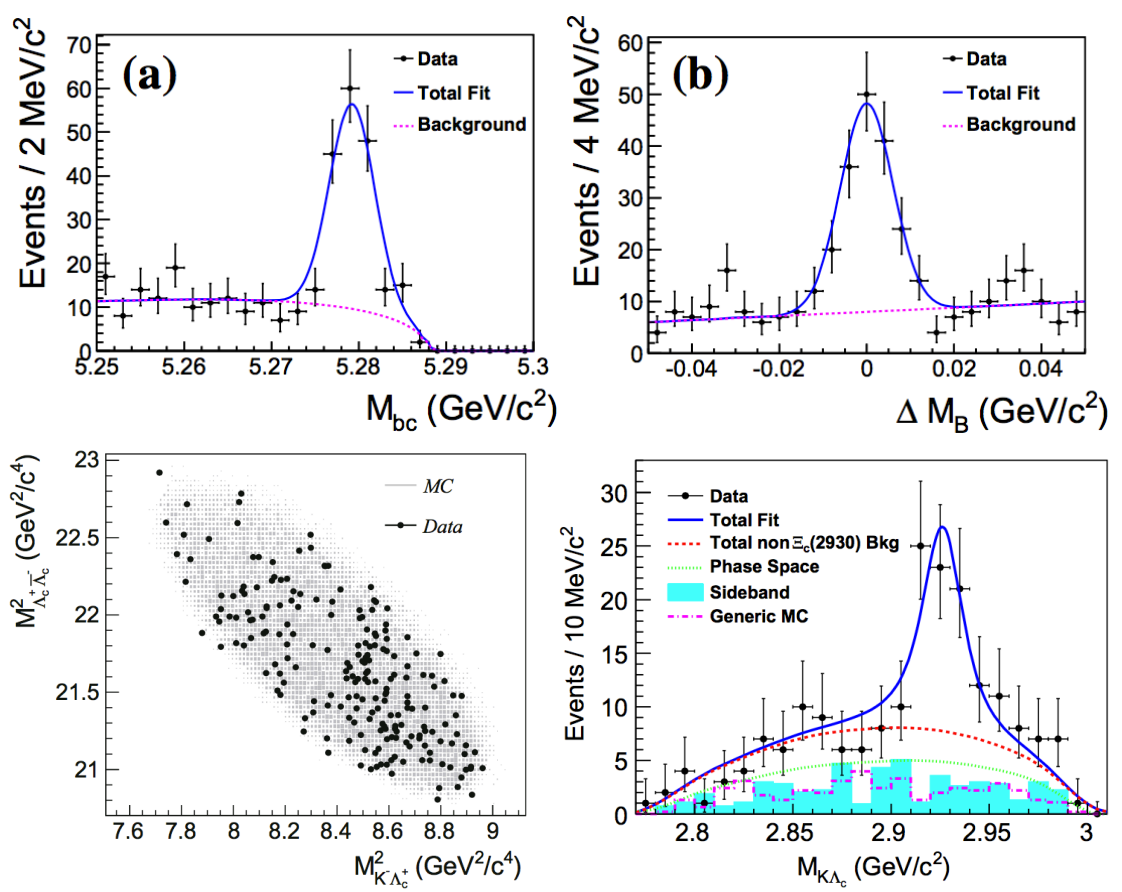

Figure 4: The distributions of (a) $M_{b c}$ and (b) $\Delta M_{B}$ in each other's signal region for $B^{-} \rightarrow K^{-} \Lambda_{c}^{+} \bar{\Lambda}_{c}^{-}$; and its Dalitz plot distribution in the $B$ signal region. The last figure is the invariant mass distribution of $K^{-} \Lambda_{c}^{+}$.

$\Omega_{c}$ hadronic decays will shed light on the dynamics of baryon weak decays. Using the full Belle dataset, with an integrated luminosity of $980 \mathrm{fb}^{-1}$, eight decay modes of $\Omega_{c}$ are measured [[13], relative to the nominal mode $\Omega^{-} \pi^{+}$, shown in Fig. [1. The precisions of branching fractions are improved by a factor of 2 for previously measured modes, and three modes are first observations. The intermediate resonances in three decays, $\Omega_{c} \rightarrow \Omega^{-} \pi^{+} \pi^{0}, \Xi^{-} K^{-} \pi^{+} \pi^{+}$and $\Xi^{0} K^{-} \pi^{+}$, are studied for the first time, and dominant sub-mode contributions are measured shown in Fig. [.
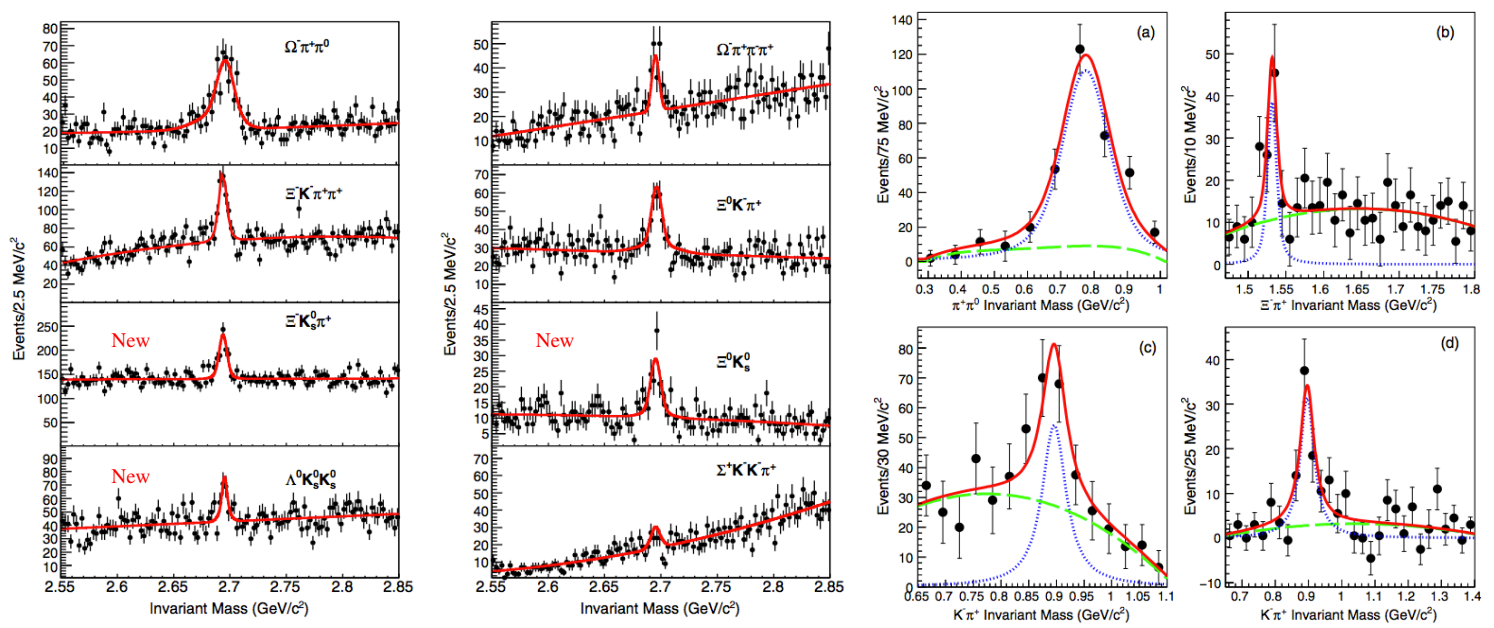

Figure 5: The invariant mass distributions for the eight decay modes in left figures. Background-subtracted invariant mass distributions for two particle combinations: (a) $\pi^{+} \pi^{0}$ for $\Omega_{c}^{0} \rightarrow \Omega^{-} \pi^{+} \pi^{0}$, (b) $\Xi^{-} \pi^{+}$and (c) $K^{-} \pi^{+}$for $\Omega_{c}^{0} \rightarrow \Xi^{-} K^{-} \pi^{+} \pi^{+}$decays, and (d) $K^{-} \pi^{+}$for $\Omega_{c}^{0} \rightarrow \Xi^{0} K^{-} \pi^{+}$decays. 


\subsection{Confirmation of excited $\Omega_{c} \rightarrow \Xi_{c}^{+} K^{-}$}

The LHCb collaboration reported the dramatic observation of five excited $\Omega_{c}$ in the invariant mass distribution of $\Xi_{c}^{+} K^{-}$by reconstructing $\Xi_{c}^{+} \rightarrow p K^{-} \pi^{+}$, based on $3.3 \mathrm{fb}^{-1}$ dataset [14]]. Using the entire Belle data sample of $980 \mathrm{fb}^{-1}$, the Belle collaboration confirms four of five narrow $\Omega_{c}$ states reported by LHCb in the same decay mode, $\Omega_{c}(3000), \Omega_{c}(3050), \Omega_{c}(3066), \Omega_{c}(3090)$ with confidence level of $3.9 \sigma, 4.6 \sigma, 7.2 \sigma$ and $5.7 \sigma$ corresponding [ㅍ]], shown in Fig. 6 .

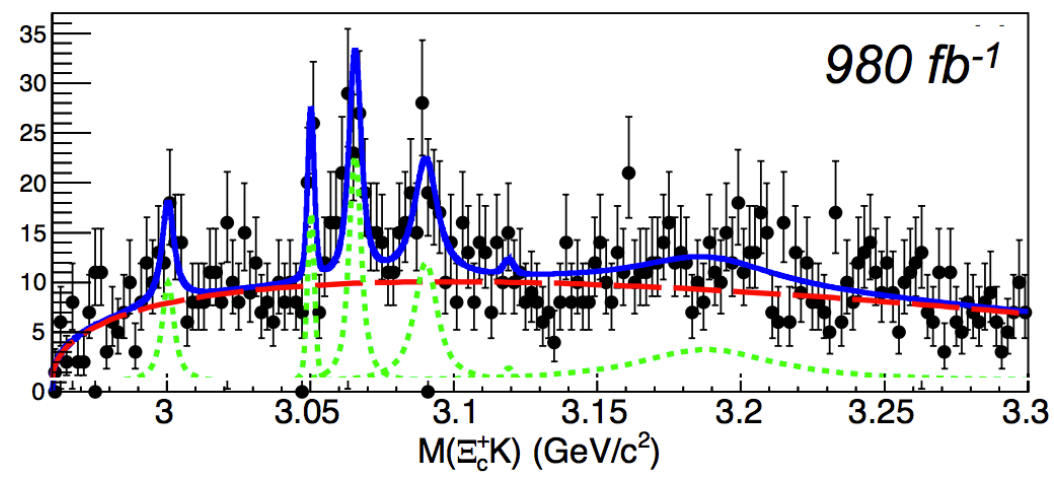

Figure 6: The invariant mass distribution of $\Xi_{c}^{+} K^{-}$with a sum of a threshold function (dashed line) and six Voigtian fucntions, with fixed masses, intrinsic widths and resolutions (dotted lines).

\subsection{Production rate of various baryons at $10.52 \mathrm{GeV}$}

Using an $800 \mathrm{fb}^{-1}$ data sample taken near the $\Upsilon(4 S)$ resonance, the inclusive production cross sections of hyperons and charmed baryons from $e^{+} e^{-}$annihilation are measured at Belle [16]]. The feed-down contributions from heavy particles are subtracted, and the direct production cross sections are presented for the first time. The production cross section divided by the number of spin states for $S=-1$ hyperons follow an exponential function with a single slope parameter except for the $\Sigma(1395)^{+}$resonance. Suppression of $\Sigma(1385)^{+}$and $\Xi(1530)^{0}$ hyperons is observed. Among the production cross sections of charmed baryons, a factor of 3 difference for $\Lambda_{c}^{+}$states over $\Sigma_{c}$ states is observed in Fig. $\square$. This observation suggests a diquark structure for these baryons.

\section{Summary}

$\mathrm{BaBar}$ and Belle have achieved fruitful results on charm physics at B-factories, based on an integrated luminosity of about $988 \mathrm{ab}^{-1}$ and $518 \mathrm{ab}^{-1}$, respectively, such as $D^{0}-\bar{D}^{0}$ mixing and $C P$ violation in many $D$ decay channels, studies on the charmed baryons decays and spectroscopy, etc. Considering a larger charm sample to be collected by Belle II at SuperKEKB, the next generator $B$-factory, let us look forward to the charming news of charm physics from Belle II.

\section{References}

[1] Ed. A.J. Bevan, B. Golob, Th. Mannel, S. Prell, and B.D. Yabsley, Eur. Phys. J. C74(2014) 3026, SLAC-PUB-15968, KEK Preprint 2014-3. 

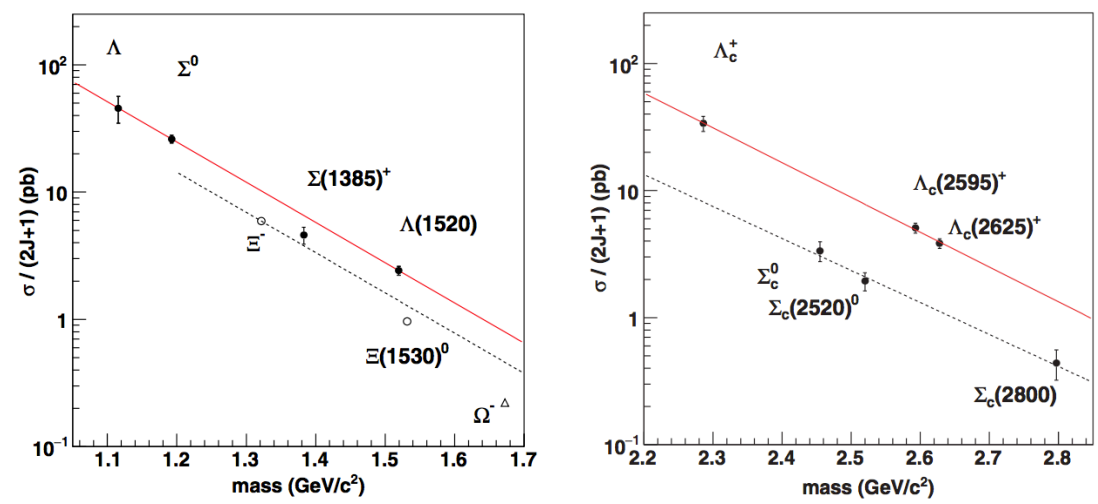

Figure 7: Scaled direct production cross section as a function of mass of hyperons (left figure) or charmed baryons (right figure).

[2] Érica Polycarpo, Alerto C. dos Reis, Review of recent results on charm mixing and $C P$ violation, Int] ए. Mod. Phys. A 29, 1430051 (2014); G. Casarosa's report at SLAC experimental seminar 2016.

[3] R. Aaij et al. (LHCb Collaboration), Phys. Rev. Lett. 110, 101802 (2013); Phys. Rev. Lett. 111, 251801(2013); T. Aaltonen et al. (CDF Collaboration), Phys. Rev. Lett. 111, 231802(2013);

[4] B. R. Ko et al. (Belle Collaboration), Phys. Rev. Lett. 112, 111801 (2014).

[5] HFLAV: Charm Physics Paramters, http://www.slac.stanford.edu/xorg/hfag/charm

[6] J.P. Lees, et al. (BaBar Collaboration), Measurement of the neutral D meson mixing parameters in a time-dependent amplitude analysis of the $D^{0} \rightarrow \pi^{+} \pi^{-} \pi^{0}$, Phys. Rev. D 93, 112014(2016).

[7] K. Prasanth, et al. (Belle Collaboration), First measurement of $T$-odd moments in $D^{0} \rightarrow K_{S}^{0} \pi^{+} \pi^{-} \pi^{0}$ decays, Phys. Rev. D 95, 091101(R) (2017).

[8] V. Babu, et al. (Belle Collaboration), Search for $C P$ violation in the $D^{+} \rightarrow \pi^{+} \pi^{0}$ decay at Belle, Phys. Rev. D97, 01101(R) (2018).

[9] B. R. Ko et al. (Belle Collaboration), Evidence for $C P$ violation in the decay $D^{+} \rightarrow K_{S}^{0} \pi^{+}$, Phys. Rev. Lett. 109, 021601(2012).

[10] B. Pal, et al. (Belle Collaboration), Search for $\Lambda_{c}^{+} \rightarrow \phi p \pi^{0}$ and branching fraction measurement of $\Lambda_{c}^{+} \rightarrow K^{-} \pi^{+} p \pi^{0}$, Phys. Rev. D96,051102(R)(2017).

[11] M. Ablikim et al. (BESIII Collaboration), Phys. Rev. Lett. 116, 052001(2016).

[12] Y.B. Li, et al. (Belle Collaboration), Observation of $\Xi_{c}(2930)^{0}$ in $B^{-} \rightarrow K^{-} \Lambda_{c}^{+} \bar{\Lambda}_{c}^{-}$, Eur. Phys. J. C78 (2018) 252.

[13] J. Yelton, et al. (Belle Collaboration), Measurement of branching fractions of hadronic decays of the $\Omega_{c}$ baryon, Phys. Rev. D 97, 032001 (2018).

[14] R. Aaij et al. (LHCb Collaboration), Observation of five new narrow $\Omega_{c}^{0}$ states decaying to $\Xi_{c}^{+} K^{-}$, Phys. Rev. Lett. 118, 182001 (2017).

[15] J. Yelton, et al. (Belle Collaboration), Observation of excited $\Omega_{c}$ charmed baryons in $e^{+} e^{-}$collisions, Phys. Rev. D 97, 051102(R) (2018).

[16] M. Niiyama, et al. (Belle Collaboration), Production cross sections of hyperons and charmed baryons from $e^{+} e^{-}$annihilation near $\sqrt{s}=10.52 \mathrm{GeV}$, Phys. Rev. D 97, 072005(2018). 